\title{
Role of FABP3 as biomarker in Alzheimer's disease and synucleinopathies
}

\author{
Federica Nicoletta Sepe ${ }^{1}$, Davide Chiasserini² \& Lucilla Parnetti*,1 \\ ${ }^{1}$ Center for Memory Disturbances, Lab of Clinical Neurochemistry, Section of Neurology, Department of Medicine, University of \\ Perugia, Perugia, Italy \\ ${ }^{2}$ Stoller Biomarker Discovery Centre, School of Medical Sciences, Faculty of Biology, Medicine and Health, The University of \\ Manchester, Manchester, United Kingdom \\ *Author for correspondence: Tel.: +39 075578 3545; lucilla.parnetti@unipg.it
}

Lipids are fundamental components of brain cells as they are involved in several essential processes like remodeling of plasma membrane, synaptic function and receptor-ligand interactions. Systemic and brain alterations in lipid metabolism have been linked to the pathogenesis of neurodegenerative disorders like dementia and parkinsonisms. Intracellular transport of lipids is regulated by fatty acid-binding proteins. Recently, a member of this family, the fatty acid-binding protein 3 has been proposed as a potential biomarker across a range of neurodegenerative diseases, including Alzheimer's disease and dementia with Lewy bodies. In this special report, we describe recent progresses in characterizing the role of fatty acid-binding protein 3 in neurodegeneration and its putative role as biomarker measurable in biological fluids.

First draft submitted: 23 February 2018; Accepted for publication: 16 May 2018; Published online: 27 September 2018

Keywords: $\alpha$-Synuclein $\bullet$ Alzheimer's disease $\bullet$ Amyloid $\beta \bullet$ CSF $\bullet$ FABP3 $\bullet$ neurodegenerative disorders $\bullet$ Parkinson's disease $\bullet$ phosphorylated tau $\bullet$ tau

\section{Lipids in CNS: introductory remarks}

Nowadays, the role of diagnostic biomarkers in the diagnosis of Alzheimer's disease (AD) is well established also in the early stages of the disease. Among the biomarkers currently used in diagnostic settings, we can distinguish biochemical and imaging biomarkers. The core biochemical markers are represented by the 42 amino acid peptide derived from the $\beta$-amyloid protein (A $\beta 42)$, the total tau protein (t-tau) and its phosphorylated form at threonine 181 (p-tau) that show a particular AD profile in cerebrospinal fluid (CSF) of AD and mild cognitive impairment (MCI) patients progressing to $\mathrm{AD}$ [1]. In addition, several radiological techniques are also used to support the clinical diagnosis of $\mathrm{AD}$, such as fluorodeoxyglucose positron emission tomography (FDG-PET), amyloid PET and MRI. Progressive cerebral atrophy (the entorhinal cortex is typically the earliest site of atrophy, closely followed by the hippocampus, amygdala, and parahippocampus) is a characteristic feature of $\mathrm{AD}$ neurodegeneration that can be visualized with structural MRI, as hypometabolism that gradually worsens in the posterior midline cortices of the parietal (precuneus) and posterior cingulate gyri, the inferior parietal lobule, posterolateral portions of the temporal lobe, as well as the hippocampus and medial temporal cortices is detectable in FDG-PET scans or amyloid deposition seen in amyloid-PET scans $[2,3]$. On the other hand, there are currently no biochemical or imaging biomarkers so well established for the early diagnosis of other neurodegenerative diseases like Parkinson's disease (PD) and PD with dementia (PDD), frontotemporal dementia (FTD), dementia with Lewy bodies (DLB) and multiple system atrophy (MSA). In this context, in most recent revision of National Institute on Aging-Alzheimer's Association guidelines (NIA-AA) criteria [1] the authors underlined the limitations of CSF 'core' AD biomarkers. The anatomopathological alteration detectable in $\mathrm{AD}$ and the other main neurodegenerative disorders with or without dementia associated were grouped in three categories, on the basis of the abnormal deposition in the brain parenchyma of one of these proteins compared with the others: amyloid deposition (A), tauopathy (T) and neurodegeneration or neuronal injury $(\mathrm{N})$. This proposal to revise the AD diagnostic criteria is based on the evidence that the postmortem examination on healthy older adults or patients with other types of dementia 
(vascular dementia $[\mathrm{VaD}], \mathrm{PDD}, \mathrm{FTD}$, etc.) identified the same amyloidosis and neuronal degeneration that are detectable in $\mathrm{AD}$ patients [4]. In this sense, researchers hypothesized a 'continuum' in neurodegeneration that linked together the clinical phenotypes expressed in old patients with dementia, so called 'late onset dementia, ILODs' [5]. This heterogeneous group included, for example, patients with late onset AD or FTD, PD patients that develop dementia several years after the onset of motor symptoms or patients with dementia that not fulfilled all diagnostic criteria for $\mathrm{AD}$. In this spectrum we should also consider asymptomatic elderly people with the evidence of $\mathrm{A} \beta$ amyloid aggregation or neurofibrillary tangles that will not develop dementia [5,6]. These limitations emphasize the need for more specific and sensitive biomarkers in both CSF and other biological fluids to possibly improve the accuracy of the early diagnosis of neurodegenerative disorders. The final goal is to improve early diagnosis and diagnostic accuracy.

Neurodegenerative disorders are recognized as multifactorial diseases and their onset is strongly associated with age [7]. Among the multiple factors associated with development of neurodegenerative diseases like $\mathrm{AD}[8,9]$, systemic or CNS-related alterations of lipids and their protein carriers seem to have a central pathogenetic role, as shown by epidemiological and molecular studies [10]. Indeed, the strongest genetic risk factor for sporadic $\mathrm{AD}$ is the presence of the apolipoprotein $\mathrm{E}(A P O E)$ epsilon $4(\varepsilon 4)$ allele, related to lipid transport and $\mathrm{A} \beta$ metabolism [11,12]. It is also interesting to note that $\alpha$-Synuclein $(\alpha-$ Syn), the main misfolding protein in PD, has been hypothesized to function as a fatty acid binding protein (FABP) in the CNS [13]. $\alpha$-Syn seems also to affect brain lipid metabolism, facilitating brain phospholipids fatty acid turnover by influencing endoplasmic reticulumlocalized lipid biosynthetic enzymes [14]. Besides apolipoproteins, FABPs, a family of intracellular lipid carriers, are currently studied for their possible role as putative neurodegeneration biomarkers. Among FABPs, FABP3 has been linked to the molecular mechanisms of neurodegeneration, being also proposed as candidate biomarker, due to its increase in the CSF of patients with $\mathrm{AD}, \mathrm{PDD}, \mathrm{DLB}$ and $\mathrm{VaD}$ [15-18]. The aim of this review is to describe the emerging role of this protein, considering: physiological and pathological interactions with amyloid plaques formation and $\alpha$-Syn oligomerization; its role as biomarker for the main neurodegenerative disorders not only in patients with dementia. Brain lipids play a fundamental role in CNS physiology, with their heterogeneous range of functions such as: formation and remodeling of cellular membranes; regulation of synaptic functions; modulation of intracellular signaling and receptor-ligand interactions [19,20]. Long-chain polyunsaturated fatty acids (PUFAs), such as docosahexaenoic acid (DHA; omega-3) and arachidonic acid (ARA; omega-6), are essential fatty acids in the mammalian brain [21]. Their inclusion in the membrane phospholipids is known to modulate the fluidity and permeability of cell membranes and contribute to the bilayer dynamics and neurite growth [22]. Furthermore, PUFAs can regulate the formation of lipid rafts, which play an important role in neurotransmission, ion channel formation and synaptic plasticity [23]. In young rats, the administration of DHA improved learning ability [24] suggesting a potential role of PUFA in memory and cognitive processes. In a transgenic AD mouse model, that exhibits both $A \beta$ and tau pathologies [25], DHA supplementation caused a significant reduction in soluble and intraneuronal $A \beta$ levels as well as tau phosphorylation [26] both in hippocampus and parietal cortex. In a neuroblastoma cell line, treatment with high DHA levels directly inhibited the amyloidogenic $\beta$ - and $\lambda$-secretase activities, resulting in a dose-dependent reduction of $A \beta$ levels and a gain of function of nonamyloidogenic amyloid precursor protein (APP) processing [27]. The intracellular transport of fatty acids, cholesterol and retinoids is facilitated by FABPs, a multigene family of small lipid-binding proteins, widely expressed in mammalian tissues [28].

\section{FABP3 in CNS \& neurodegeneration}

Three types of FABPs are mainly expressed in the CNS: FABP3 (heart-type FABP) has a preference for binding omega-6 PUFA; FABP5 (epidermal-type FABP), which binds saturated fatty acids; and FABP7 (brain-type FABP), with a preference for omega-3 PUFA [16]. The principal functions of FABPs in the CNS are being elucidated by a combination of gene knockout mouse models, cell culture studies, molecular analyses of gene regulation and the examination of gene polymorphisms and their physiological correlates [17-19]. In CNS, they were first purified in rodents as a protein fraction of low molecular weight capable of binding labeled oleic acid [29]. This protein was later revealed to be identical to FABP3 in humans [30]. The specific role of FABPs, in particular of FAPB3, in transporting PUFAs, might link their role as lipid transporters to the regulation of membrane composition and fluidity, and to the pathogenesis of principal neurodegenerative disorders, [31] as summarized in Figure 1.

In the CNS, FABP3 mRNA is barely detectable during embryonic development and it gradually increases after birth until adulthood [16-19]. In the adult brain FABP3 is mostly expressed in the gray matter of olfactory mitral cell layer, cerebral neocortex (layers II-VI) and hippocampal CA1 and CA2 portions [16,17], where it may play a role in 


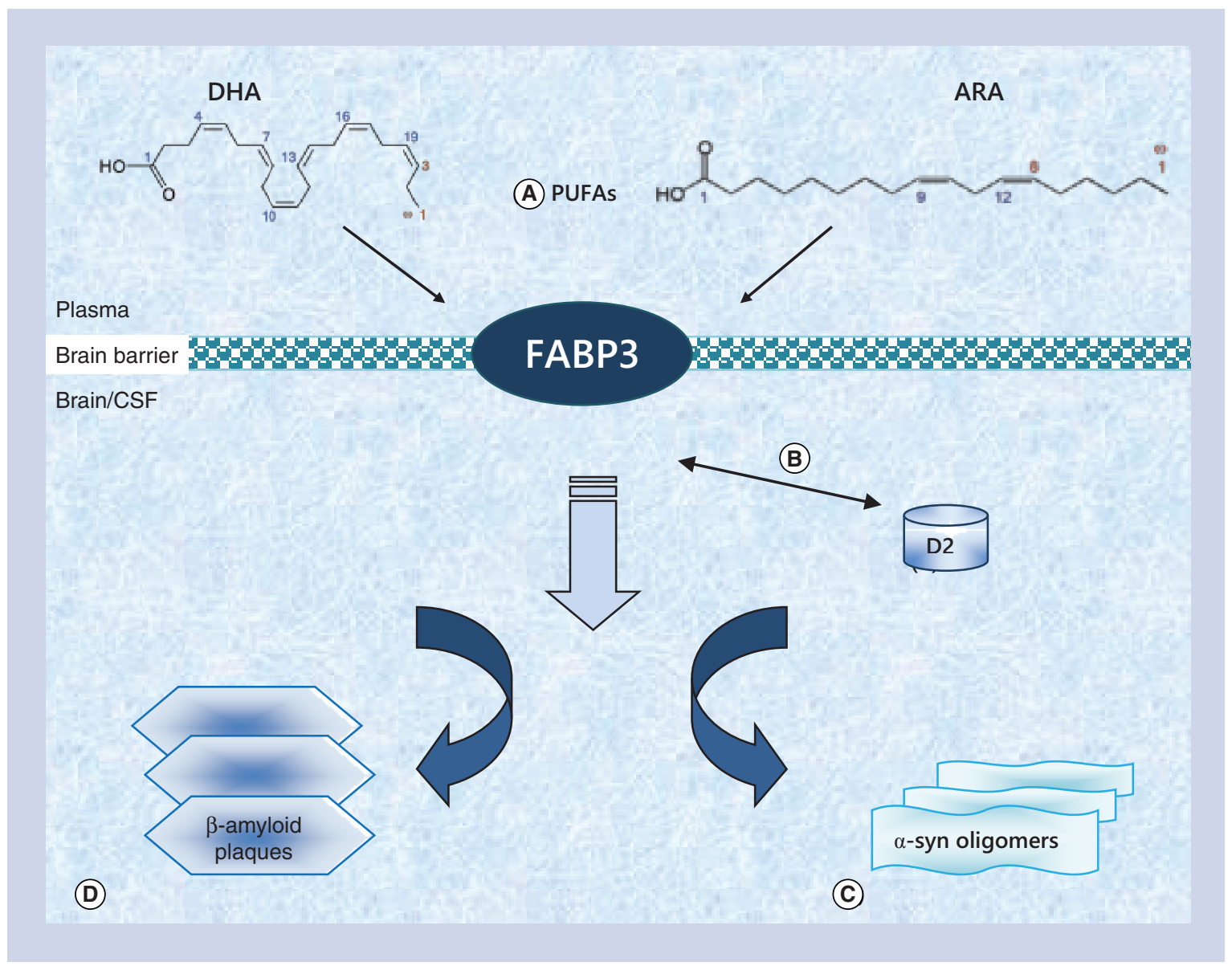

Figure 1. FABP3 roles. FABP3 belongs to the multigene family of fatty acid binding proteins that work as protein carriers of polyunsaturated fatty acids. (A) In brain the most abundant polyunsaturated fatty acids are docosahexaenoic acid (on the left) and arachidonic acid (on the right) mostly transported by this protein. FABP3 seems to be not just involved in lipid transport in the brain, but it seems to play a pivotal role in neurodegeneration. (B) In dopaminergic neurons FABP3 interacts with the D2 receptor and may modulate emotional responses. (C) FABP3 could also facilitate $\alpha$-Synuclein oligomerization. (D) FABP3 seems to be implicated indirectly in early formation of $\beta$-amyloid plaques by its interaction with lipids and its role in modulating lipid rafts (more details and definitions in the text).

neuronal synapse formation [17-19]. FABP3 physiological role in CNS as a PUFAs binding protein has been studied using FABP3 null mice. Lack of FABP3 expression induced a reduced phospholipids mass in brain, compared with the wild-type phenotype. Interestingly, a different effect was noticed for the incorporation of palmitic and ARA in phospholipids by FABP3 ablation, while palmitic acid incorporation was unchanged, showing that FABP3 may be specifically involved in $\mathrm{n}-6$ fatty acid transport/metabolism, not compensated by expression of other FABPs [25]. ARA is quantitatively one of the most common PUFAs in brain and ARA knockout mice display hippocampal and cortical atrophy [32]. Furthermore, ARA and indirectly FABP3 as its crucial lipid carrier may also have a role in amyloid plaques formation, neuroinflammation and NDMA receptor synaptic signaling [32].

The role of FABP3 in neurodegeneration seems not only limited to AD but may be also related to $\alpha$-Syn accumulation pathways and deregulation of the dopaminergic pathway in synucleinopathies. Using yeast twohybrid system with a mouse brain cDNA library, FABP3 was shown to be co-localized to the D2 receptor (D2R) positive cells, where it seems to interact with a long isoform of this receptor, previously associated with several neuropsychiatric disorders [33]. Dopamine D2 receptor is a G-protein-linked receptor expressed mainly in the striatum, but also in many other brain regions, including cerebral cortex, globus pallidus, substantia nigra and nucleus accumbens that can exist in either a state of high affinity for dopamine, D2High, or in a state of low affinity for dopamine, D2Low [34]. It seems to be involved in several biological processes including emotional response, development of psychosis in schizophrenia and other psychiatric disease and the sensitivity to dopaminergic therapy 
in PD patients [34]. The interaction between FABP3 and D2R, together with the association between a reduction of dopamine binding density to D2R and PUFAs deficiency in rodents, may suggest that FABP3 could regulate emotional responses in brain via the cellular uptake of fatty acid and the interaction with D2R [35]. It is also interesting to note that $\alpha$-Syn has been hypothesized to function as a fatty acid binding protein in the CNS [36]. Like FABPs, $\alpha$-Syn is a small cytosolic protein that binds fatty acids with high affinity in vitro. It seems to also affect brain lipid metabolism, facilitating brain phospholipids fatty acid turnover by influencing endoplasmic reticulum-localized lipid biosynthetic enzymes [37]. More direct evidence for this hypothesis is provided by a study that demonstrated that in $\alpha$-Syn-deficient astrocytes, the cellular lipid pool is altered by reducing the uptake of 16:0 fatty acids, similarly to the effect of FABP3 in cell cultures [38].

\section{FABP3 \& $A D$}

$\mathrm{AD}$ is a multifactorial disease, characterized by several intertwining pathological processes, including, but not limited to, the presence of neurofibrillary tangles and neuritic plaques, hypomyelination, neuroinflammation and cholinergic dysfunction [38,39]. Given the insufficient knowledge about pathological pathways in sporadic AD, new tools are required to identify the molecular changes underlying the disease. Levels of specific secreted cellular proteins in CSF or plasma may correlate with pathological changes in the AD brain and can thus be used to discover altered pathways linked to this disorder. Genetic and pathological studies underlie a key role for A $\beta$ peptide and tau hyperphosphorylation in AD. The pathological findings in the brain are mirrored in CSF, where levels of soluble A $\beta$ peptide 1-42 (AB42) are decreased in AD CSF, and this reduction has been linked to A 42 deposition into amyloid plaques in the brain parenchyma [40]. On the other hand, the protein tau ( $\mathrm{t}$-tau) and its phosphorylated form at threonine 181 ( $\mathrm{p}$-tau181) are increased in the CSF of AD patients. These three markers are currently used to support the clinical diagnosis of $\mathrm{AD}$ and have also shown a good performance in detecting early $\mathrm{AD}$ [41]. Moreover, ratios between the concentration of $\mathrm{p}$-tau181 and total tau (t-tau) or A $\mathrm{B} 42$ are predictive of future development of $\mathrm{AD}$ in patients with MCI [42] and cognitive decline [43] or brain atrophy in nondemented older adults [44]. Despite the usefulness of these CSF biomarkers in $\mathrm{AD}$ diagnosis, they provide little information on pathways other than those amyloid and tau related. Some members of the complex molecular networks involved in neurodegeneration, such as cytokines, chemokines, lipid-binding proteins or growth factors, have been reported to have different expression levels in patients with neurological diseases compared healthy controls but without a peculiar expression paradigm that could be useful in clinical routine diagnosis [45]. These limitations have stimulated the application of 'omics' technologies in AD, measuring a large numbers of analytes in plasma and CSF, including FABP3, with the goals of defining disease biomarkers and uncovering potential points of pharmacological intervention [46]. Based on this model, a 2010 study measured ten different analytes, including FAPB3, both in plasma and CSF of 20 AD patients and 20 cognitively healthy controls. CSF levels of FABP3 were elevated in patients with AD, those with incipient $\mathrm{AD}$, and those with clinical $\mathrm{AD}$ and concomitant cerebrovascular disease compared with control subjects $(\mathrm{p}<0.01)$ [47]. Another work showed that CSF FABP3 concentration distinguished between healthy controls and patients with $\mathrm{AD}$ with a sensitivity and specificity of 76 and $84 \%$, respectively. Both patients with $\mathrm{AD}$ and $\mathrm{MCI}$ due to AD had higher FABP3 levels in CSF when compared with cognitively healthy controls [48]. Notably, FABP3 diagnostic performance is significantly lower as compared with A $\beta 42$, $t$-tau and p-tau 181 (at least $85 \%$ sensitivity and $80 \%$ specificity) and represents the huge limitation in its current use in clinical practice [49]. In the same year we analyzed the correlation between FABP3 CSF levels and A $\beta 42$, $t$-tau and $p$-tau in aroup of patients with MCI $(\mathrm{n}=41), \mathrm{AD}(\mathrm{n}=32)$ and subjects with other neurological diseases without dementia $(\mathrm{OND}, \mathrm{n}=25)$ followed up for 4 years. FABP3 levels were higher in $\mathrm{AD}$ and in $\mathrm{MCI}$ converting to $\mathrm{AD}(\mathrm{MCI} / \mathrm{AD})$ with respect to $\mathrm{OND}$ and to cognitively stable MCI patients (MCI/MCI), showing a sensitivity of $87 \%$ and a specificity of $81 \%$ for $A D$ versus $\mathrm{OND}$, and 46 and 94\%, when comparing MCI/MCI versus MCI/AD, respectively. FABP3 correlated strongly with both $\mathrm{t}$-tau and $\mathrm{p}$-tau possibly showing the role of $\mathrm{FABP} 3$ as neurodegeneration biomarker. FABP3/AB 42 ratio improved the performance in distinguishing both $\mathrm{AD}$ from $\mathrm{OND}$ and $\mathrm{MCI} / \mathrm{AD}$ from $\mathrm{MCI} / \mathrm{MCI}$. Additionally, a significant correlation between FABP3/A 42 ratio and Mini Mental State Examination (MMSE) annual decrease rate was documented [50].

Also Harari, in 2014, found that the FABP3/A 42 ratio demonstrates a similar hazard ratio to p-tau/A 342 , for disease conversion suggesting that FABP3 may be an independent prognostic factor, increasing at very early stages of the disease [51]. Recently, another study confirmed this hypothesis [52]. This work examined the CSF levels of FABP3 as a biomarker for development of dementia in 86 nondemented women aged 70-84 years, who participated in the Prospective Population Study of Women in Gothenburg for 8 years and its link with A $\beta 42$, 
t-tau, p-tau and CSF/serum albumin ratio. Between 1992 and 2000, eight women developed dementia (four $\mathrm{AD}$, three $\mathrm{VaD}$, one mixed $\mathrm{VaD}$ and $\mathrm{AD}$ ). Higher CSF FABP3 levels at baseline were related to development of dementia and in particular AD during follow-up. FABP3 highly correlated with CSF t-tau, p-tau and CSF/serum albumin ratio, but not with $\mathrm{A} \beta 42$. FAPB3 also seems to be related with entorhinal cortex atrophy and $A P O E \varepsilon 4$ carrier status, as demonstrated by Desikan and co-authors. Using linear mixed effects models, they measured CSF FABP3 levels in a cohort of 90 cognitively normal healthy older adults (healthy controls [HC]), 139 individuals diagnosed with amnestic MCI and 66 with probable AD from the Alzheimer's disease Neuroimaging Initiative. In this a multiplex-based immunoassay panel, co-varied for age, sex, presence ('carriers') or absence ('noncarriers') of at least one $\varepsilon 4$ allele of $A P O E$ ( $A P O E \varepsilon 4$ ), diagnostic status (AD vs MCI vs cognitively normal older adults) and disease severity (Clinical Dementia Rating Scale score at baseline) at baseline, FABP3 levels strongly correlates with $A P O E(\beta$-coefficient $=0.86, \mathrm{p}=2.9 \times 10-16)$ as with cortex atrophy in regions of interest for $\mathrm{AD}$, irrespectively of p-tau status [53].

Considered together, these results suggest a potentially direct role for fatty acid binding proteins in early AD pathobiology, reflecting CNS lipid dyshomeostasis.

\section{FABP3 \& synucleinopathies}

$\alpha$-Syn is the major component of Lewy bodies, the typical intracellular inclusions of PD and other neurodegenerative disorders like DLB, PDD and MSA. Collectively they are so called synucleinopathies. This 140 -amino acid protein is associated with synaptic vesicles in presynaptic nerve terminals [54], and its accumulation is linked to the progressive loss of dopaminergic (DA) neurons, a main feature of PD pathogenesis [55]. $\alpha$-Syn toxicity is triggered by oligomerization of $\alpha$-Syn in vitro [56] and in vivo [57], indicating that oligomerization underlies cytotoxic events in PD. However, mechanisms underlying $\alpha$-Syn oligomerization are unclear. $\alpha$-Syn, similarly to FABP3, can bind fatty acids, particularly PUFAs [58,59], and its oligomerization in cultured mesencephalic neuronal cells seems to be enhanced by exposure to this kind of fatty acids. Abnormally high PUFAs levels are observed in $\alpha$-Syn-transfected mesencephalic neuronal cells and in postmortem examination PD brains, whereas lower levels are expressed in mice lacking $\alpha$-Syn [59]. All together these results suggest that PUFAs binding to $\alpha$-Syn is a key event in generating pathogenic $\alpha$-Syn oligomers. The link between FABP3 and $\alpha$-Syn is further shown by the high expression of FABP3 in dopaminergic neurons in the substantia nigra pars compacta [60]. FABP3 overexpression seems to accelerate arachidonic acid-induced $\alpha$-Syn oligomerization promoting apoptosis in animal models [61]. In human CSF, $\alpha$-Syn levels are typically lower in PD than in controls, while in AD its concentration is increased and correlates with $t$-tau, suggesting that $\alpha$-Syn may also be a nonspecific biomarker for DA system degeneration [62]. However, the performance of $\alpha$-Syn as PD biomarker is currently below the acceptance criteria for its introduction in clinical practice [63], therefore, new and more sensitive biomarkers are needed to improve the diagnosis of PD and other synucleinopathies [64]. Several studies have investigated the role of FABP3 as fluid biomarkers in synucleinopathies, both in CSF and blood. A paper published in 2007 established, in a cohort of patients with $\operatorname{DLB}(\mathrm{n}=33)$, PDD $(\mathrm{n}=25)$ and AD $(\mathrm{n}=35)$, that serum levels of FABP3 significantly differed between DLB and $\mathrm{AD}$, and PDD and DLB [65]. A coeval research confirmed this hypothesis [66]. The authors measured serum levels of FABP3 in patients with DLB, PD and AD using a solid-phase enzyme-linked immunoassay. Iodine-123 metaiodobenzylguanidine cardiac scintigraphy was performed on each patient and the heart to mediastinum $(\mathrm{H} / \mathrm{M})$ ratio was also calculated. Serum levels of FABP3 were significantly higher in DLB patients and PD patients than in $\mathrm{AD}$ ones. $\mathrm{H} / \mathrm{M}$ ratios of the DLB and PD patients were significantly lower than AD ones. The diagnostic value of the serum FABP3 levels between AD and DLB patients was inferior to that of the delayed H/M ratio of iodine-123 metaiodobenzylguanidine cardiac scintigraphy. Multivariate regression analysis revealed that the delayed $\mathrm{H} / \mathrm{M}$ ratio predicted serum FABP3 levels in the PD patients. This work demonstrated that the increase of serum FABP3 in Lewy body disease patients may be associated with cardiac sympathetic nerve dysfunction.

Interestingly, a 2011 study measured FABP3 in serum samples from patients with either AD ( $\mathrm{n}=31)$, PD, $(n=43)$ or other cognitive disorders $(n=42)$ and in 52 healthy controls. FABP3 serum levels were not different among the diagnostic groups and only correlated positively with age, without any apparent clinical significance [67]. Likewise for AD, CSF FABP3 levels seem to be predictive for developing dementia in patients with PD and MCI-PD as demonstrated by a regional population-based, prospective cohort study involving 128 nondemented patients with new-onset parkinsonism (104 with PD, 11 with MSA and 13 with progressive supranuclear palsy, PSP) who were followed up for 5-9 years [68]. CSF levels of neurofilament light chain protein, A $\beta 42$, t-tau, p-tau, $\alpha$-Syn and FABP3 were quantified at baseline and after 1 year and compared with the CSF results from 30 healthy 
control participants. In PD, high neurofilament light chain protein, low A $\beta 42$ and high FABP3 at baseline were significantly associated with the development of dementia. This peculiar CSF pattern seemed to be also useful in discriminating between patients with PSP and controls. Recently, our group performed a prospective study in a large cohort of patients $(n=208)$, to evaluate CSF FABP3 performance in differentiating between AD, PDD, DLB, $\mathrm{PD}$ and neurological controls. FABP3 levels were significantly increased in patients with $\mathrm{AD}$ and DLB compared with those with PD and controls. Additionally, the combination of FABP3 with p-tau showed high accuracy in differentiating between $\mathrm{AD}$ and $\mathrm{DLB}$, while the combination of $\mathrm{p}$-tau, FABP3 and $\alpha$-Syn better discriminated between $\mathrm{AD}$ and $\mathrm{PDD}$ patients [69]. This two different studies partially differ for the population selected (MCI/PD vs PDD, and PSP patients and AD patients vs DLB, PDD and PD ones), but agree about the promising role of FABP3 as a predictor of dementia and its correlation with cognitive decline (measured by MMSE score at follow-up).

\section{Conclusions}

Despite some limitations for its use in clinical practice (it is currently difficult to estimate FABP3 diagnostic value in plasma due to its higher expression in peripherical tissues, especially heart and CNS; further investigations are needed to validate its role in atypical parkinsonisms and neurodegeneration) FABP3 has been proposed as an unspecific biomarker of neurodegeneration, but not as a specific disease-related marker. In fact, it is not completely clear whether FABP3 - which has been found to be increased in serum and/or CSF of Creutzfeld-Jakob disease, traumatic brain injury, ischemic stroke and subarachnoid hemorrhage [14] - represents a marker of neuronal dysfunction or rather reflects cell death. This findings may imply that FABP3 may be released from brain tissue into CSF after cell death, or alternatively that FABP3 alone may increase neuron vulnerability to oxidative stress, as seen in DA neurons during $\alpha$-Syn oligomerization [59] or at early stages of amyloid plaques formation.

\section{Future perspective}

CSF FABP3 represents a promising biomarker for use in clinical practice and several studies suggest that it will be considered not only a predictive factor for progression to dementia in different neurological disorders, but also prove useful for differential diagnosis between neurodegenerative diseases, in combination with other CSF biomarkers.

\section{Executive summary}

FABP3 in CNS \& neurodegeneration

- FABP3 interferes with the lipid composition, fluidity and function of the cell membrane, being related to $A \beta$ levels and $\alpha$-Synuclein pathological alterations. Further studies are needed to establish the functional interaction between FABP3 and tau.

- Cerebrospinal fluid (CSF) FABP3 are increased in patients with Alzheimer's disease (AD), Parkinson's disease (PD) with dementia, dementia with Lewy bodies (DLB) and also in vascular dementia.

FABP3 \& Alzheimer's disease

- CSF FABP3 levels are increased at early phases of AD and PD, and is associated with the onset of cognitive deterioration.

- CSF FABP3 levels are significantly increased both in AD and DLB, as compared with PD; the combination with other biomarkers shows high accuracy in differentiating $A D$ from DLB.

FABP3 \& synucleinopathies

- Serum FABP3 levels may discriminate PD versus other parkinsonisms with dementia and AD.

Financial \& competing interests disclosure

The authors have no relevant affiliations or financial involvement with any organization or entity with a financial interest in or financial conflict with the subject matter or materials discussed in the manuscript. This includes employment, consultancies, honoraria, stock ownership or options, expert testimony, grants or patents received or pending, or royalties.

No writing assistance was utilized in the production of this manuscript. 


\section{References}

Papers of special note have been highlighted as: • of interest; $\bullet \bullet$ of considerable interest

1. Jack CJ, Bennett DA, Blennow K et al. 2018 NIA-AA research framework to investigate the Alzheimer's disease continuum. Alzheimers Dement. 14(4), 535-562 (2018).

2. Johnson KA, Fox NC, Sperling RA et al. Brain imaging in Alzheimer disease. Cold Spring Harb. Perspect. Med. doi:10.1101/cshperspect.a006213 (2012) (Epub ahead of print).

3. Villemagne VL, Doré V, Burnham SC et al. Imaging tau and amyloid- $\beta$ proteinopathies in Alzheimer disease and other conditions. Nature reviews doi:10.1038/nrneurol.2018.9 (2018) (Epub ahead of print).

4. Rosén C, Hansson O, Blennow K et al. Fluid biomarkers in Alzheimer’s disease - current concepts. Mol. Neurodegener. 8, 20 (2013).

5. Mattson MP. Late-onset dementia: a mosaic of prototypical pathologies modifiable by diet and lifestyle. NPJ Aging Mech. Dis. doi:10.1038/npjamd.2015.3 (2015) (Epub ahead of print).

6. Jack C, R Jr, Knopman DS, Weigand SD et al. An operational approach to National Institute on Aging-Alzheimer's Association criteria for preclinical Alzheimer disease. Ann. Neurolo. doi:10.1002/ana.22628 (2012) (Epub ahead of print).

7. Jack CJ, Wiste HJ, Knopman D Vemuri et al. Rates of $\beta$-amyloid accumulation are independent of hippocampal neurodegeneration. Neurology 82, 1605-1612 (2014).

8. Okonkwo OC, Alosco ML, Griffith HR et al. Cerebrospinal fluid abnormalities and rate of decline in everyday function across the dementia spectrum: normal aging, mild cognitive impairment, and Alzheimer disease. Arch. Neurol. 67, 688-696 (2010).

9. Steinman L. Nuanced roles of cytokines in three major human brain disorders. J. Clin. Invest. 118, 3557-3563 (2008).

10. Perry VH, Cunningham C, Holmes C. Systemic infections and inflammation affect chronic neurodegeneration. Nat. Rev. Immunol. 7 , 161-167 (2007).

11. Reitz C. Dyslipidemia and the risk of Alzheimer's disease. Curr. Atheroscler. Res. 15, 307 (2013).

- Remarkably analyzes the interaction between alteration in lipids metabolism and risk of Alzheimer's disease development, and represents for us a source of inspiration for this review.

12. Walter J, van Echten-Deckert G. Cross-talk of membrane lipids and Alzheimer-related proteins. Mol. Neurodegener. 8, 34 (2013).

13. Soderberg M, Edlund C, Kristensson $\mathrm{K}$ et al. Fatty acid composition of brain phospholipids in aging and in Alzheimer's disease. Lipids. 26 (6), 421-425 (1991).

14. Bjerke M, Zetterberg H, Edman A et al. Cerebrospinal fluid matrix metalloproteinases and tissue inhibitor of metalloproteinases in combination with subcortical and cortical biomarkers in vascular dementia and Alzheimer's disease. J. Alzheimers Dis. 27, 665-676 (2011).

15. Tanaka T, Iwawaki $\mathrm{D}$, Sakamoto $\mathrm{M}$ et al. Mechanisms of accumulation of arachidonate in phosphatidylinositol in yellowtail. A comparative study of acylation systems of phospholipids in rat and the fish species Seriola quinqueradiata. Eur. J. Biochem. 270, 1466-1473 (2003).

16. Moullé VSF, Cansell C, Luquetand S et al. The multiple roles of fatty acid handling proteins in brain. Front. Physiol. 3, 285 (2012).

17. Storch J, Corsico B. The emerging functions and mechanisms of mammalian fatty acid-binding proteins. Annu. Rev. Nutr. 28, 73-95 (2008).

- Analyzes the heterogeneous biological and pathophysiological role of all fatty acid-binding proteins (FABPs) in human tissues and is useful to elaborate on the complexity of these proteins.

18. Owada Y. Fatty acid binding protein: localization and functional significance in the brain. Tohoku J. Exp. Med. 214, 213-220 (2008)

- A key work in the description of localizations and functions of FAPBs in CNS, including FABP3.

19. Storch J, Thumser AE. The fatty acid transport function of fatty acid-binding proteins. Biochim. Biochim. Biophys. Acta. 1486, 28-44 (2000).

20. Zimmerman AW, Veerkamp JH. New insights into the structure and function of fatty acid-binding proteins. Cell. Mol. Life Sci. 59, 1096-1116 (2002).

21. Veerkamp JH, Maatman RG. Cytoplasmic fatty acid-binding proteins: their structure and genes. Prog. Lipid Res. 34, 17-52 (2001).

22. Wassall SR, Stillwell W. Polyunsaturated fatty acid cholesterol interactions: domain formation in membranes. Biochim. Biophys. Acta. 1788 (1), 24-32 (2009).

23. Janssen CI, Kiliaan AJ. Long-chain polyunsaturated fatty acids (LCPUFA) from genesis to senescence: the influence of LCPUFA on neural development, aging, and neurodegeneration. Prog. Lipid Res. 53, 1-17 (2014).

24. Gamoh S, Hashimoto M, Sugioka K. Chronic administration of docosahexaenoic acid improves reference memoryrelated learning ability in young rats. Neuroscience 93(1), 237-241 (1999).

25. Oddo S, Caccamo A, Shepherd JD. Triple-transgenic model of Alzheimer's disease with plaques and tangles: intracellular A $\beta$ and synaptic dysfunction. Neuron 39(3), 409-421 (2003). 
26. Grimm MW, Zimmer VC, Lehmann J et al. The impact of cholesterol, DHA, and sphingolipids on Alzheimer's disease. BioMed. Res. Int. doi:10.1155/2013/814390 (2013) (Epub ahead of print).

27. Lautner R, Palmqvist S, Mattsson N et al. Alzheimer's disease neuroimaging I apolipoprotein E genotype and the diagnostic accuracy of cerebrospinal fluid biomarkers for Alzheimer disease. JAMA Psychiatry 71, 1183-1191 (2014).

28. Wood LP. Lipidomics of Alzheimer's disease: current status. Alzheimers Res. Ther. 4, 5 (2012).

29. Bass NM, Raghuphaty E, Rhoads DE et al. Partial purification of molecular weight 12000 fatty acid binding protein from rat brain and their effect on synaptosomal $\mathrm{Na}+$-dependent amino acid uptake. Biochemistry 23, 6539-6544 (1984).

30. Murphy EJ, Owada Y, Kitanaka $\mathrm{N}$ et al. Brain arachidonic acid incorporation is decreased in heart fatty acid-binding protein gene-ablated mice. Biochemistry 44, 6350-6360 (2005).

31. Liu RZ, Mita R, Beaulieu M et al. Fatty acid binding proteins in brain development and disease. Int. J. Dev. Biol. 54, 1229-1239 (2010).

32. Thomas M, Pelleieux S, Vitale $\mathrm{N}$ et al. Dietary arachidonic acid as a risk factor for age-associated neurodegenerative diseases: potential mechanisms. Biochimie 1, 10 (2016).

33. Takeuki Y, Fukunaga K. Differential subcellular localization of two dopamine D2 receptor isoformos in tranfected NG108-15 cells. J. Neurochem. 85, 1064-1074 (2003).

34. Seeman P. Are dopamine D2 receptors out of control in psychosis? Prog. Neuropsychopharmacol. Biol. Psychiatry 46, 146-152 (2013).

35. Sharon R, Goldberg MS, Bar-Josef I et al. $\alpha$-Synuclein occurs in lipid-rich high molecular weight complexes, binds fatty acids, and shows homology to the fatty acid-binding proteins. Proc. Natl Acad. Sci. USA 98, 9110-9115 (2001).

36. Maroteaux L, Campanelli JT, Scheller RH. Synuclein: a neuron-specific protein localized in the nucleus and presynaptic nerve terminal, J. Neurosci. 8, 2804-2815 (1988).

37. Castagnet PI, Golovko MY, Barcelo'-Coblijn GC et al. Fatty acid incorporation is decreased in atrocities cultured from $\alpha$-synuclein gene-ablated mice. J. Neurochem. 94(3), 839-849 (2005).

38. Sharon R, Bar-Joseph I, Frosch MP et al. The formation of highly soluble oligomers of $\alpha$-synuclein is regulated by fatty acids and enhanced in Parkinson's disease. Neuron 37, 583-595 (2003).

39. Dubois B, Feldman HH, Jacova C et al. Revising the definition of Alzheimer's disease: a new lexicon. Lancet Neurol. 9, 1118-1127 (2010).

40. Dubois B, Feldman HH, Jacova C et al. Research criteria for the diagnosis of Alzheimer's disease: revising the NINCDS-ADRDA criteria. Lancet Neurol. 6, 734-746 (2007).

41. Li G, Sokal I, Quinn JF, Leverenz JB et al. CSF tau/A 42 ratio for increased risk of mild cognitive impairment: a follow-up study. Neurology. 69, 631-639 (2007).

42. De Meyer G, Shapiro F, Vanderstichele H et al. Diagnosis independent Alzheimer disease biomarker signature in cognitively normal elderly people. Arch. Neurol. 67, 949-956 (2010).

43. Visser PJ, Verhey F, Knol DL et al. Prevalence and prognostic value of CSF markers of Alzheimer's disease pathology in patients with subjective cognitive impairment or mild cognitive impairment in the DESCRIPA study: a prospective cohort study. Lancet Neurol. 8, 619-627 (2009).

44. Fagan AM, Roe CM, Xiong C et al. Cerebrospinal fluid tau/ $\beta$-amyloid (42) ratio as a prediction of cognitive decline in nondemented older adults. Arch. Neurol. 64, 343-349 (2007).

45. Lewczuk P, Riederer P, O’Bryant SE et al. Cerebrospinal fluid and blood biomarkers for neurodegenerative dementias: an update of the Consensus of the Task Force on Biological Markers in Psychiatry of the World Federation of Societies of Biological Psychiatry. World J. Biol. Psychiatry doi:10.1080/15622975.2017.1375556 (2018) (Epub ahead of print).

46. Britschgi M, Rufibach K, Bauer Huang SL et al. Modeling of pathological traits in Alzheimer's disease based on systemic extracellular signalling proteome. Mol. Cell. Proteomics 10, 1-11 (2011).

47. Rosén C, Mattsson N, Johansson PM et al. Discriminatory analysis of biochip-derived protein patterns in CSF and plasma in neurodegenerative diseases. Front. Aging Neurosci. 3-1 (2011).

48. Hu WT, Chen-Plotkin A, Arnold SE et al. Novel CSF biomarkers for Alzheimer's disease and mild cognitive impairment. Acta Neuropathol. 119, 669-678 (2010).

49. Shaw LM, Vanderstichele H, Knapik-Czajka M et al. Qualification of the analytical and clinical performance of CSF biomarker analyses in ADNI. Acta Neuropathol. 121(5), 597-609 (2011).

50. Chiasserini D, Parnetti L, Andreasson U et al. CSF levels of heart fatty acid binding protein are altered during early phases of Alzheimer's disease. J. Alzheimers Dis. 22, 1281-1288 (2010).

-. One of the first works on the emerging role of FABP3 in Alzheimer's disease and mild cognitive impairment.

51. Harari O, Cruchaga C, Kauwe JSK, A et al. Ptau-A 342 ratio as a continuous trait for biomarker discovery for early stage Alzheimer's disease in multiplex immunoassay panels of cerebrospinal fluid. Biol. Psychiatry 75(9), 723-731 (2014).

-. The observation study confirms in a large court of patients the predicting role of FABP3 as biomarker. 
52. Bjerkeb M, Kerna S, Blennow K et al. Cerebrospinal fluid fatty acid-binding protein 3 is related to dementia development in a population-based sample of older adult women followed for 8 years. J. Alzheimers Dis. 49, 733-741 (2016).

53. Desikan R, Thompson WK, Holland $\mathrm{D}$ et al. Heart fatty acid binding protein and A $\beta$-associated Alzheimer's neurodegeneration. Mol. Neurodegen. 8, 39 (2013).

-• Remarkably correlates FABP3 with apolipoprotein E status and entorhinal cortex atrophy in Alzheimer's disease patients.

54. Maroteaux L, Campanelli JT, Scheller RH. Synuclein: a neuron-specific protein localized to the nucleus and presynaptic nerve terminal. J. Neurosci. 8, 2804-2815 (1988).

55. Spillantini MG, Crowther RA, Jakes R et al. $\alpha$-Synuclein in filamentous inclusions of Lewy bodies from Parkinson's disease and dementia with lewy bodies. Proc. Natl Acad. Sci. USA 95, 6469-6473 (1998).

56. Conway KA, Harper JD, Lansbury PT. Accelerated in vitro fibril formation by a mutant $\alpha$-synuclein linked to early onset Parkinson disease. Nat. Med. 4, 1318-1320 (1998).

57. Winner B, Jappelli R, Maji SK et al. In vivo demonstration that $\alpha$-synuclein oligomers are toxic. Proc. Natl Acad. Sci. USA 108, 4194-4199 (2011).

58. Mollenhauer B, El-Agnaf OM, Marcus $\mathrm{K}$ et al. Quantification of $\alpha$-synuclein in cerebrospinal fluid as a biomarker candidate: review of the literature and considerations for future studies. Biomark. Med. 4, 683-699 (2010).

59. Suzuki M, Sango K, Wada K, Nagai Y. Pathological role of lipid interaction with $\alpha$-synuclein in Parkinson's disease. Neurochem. Int. doi:10.1016/j.neuint.2017.12.014 (2018) (Epub ahead of print).

60. Sharon R, Bar-Joseph I, Mirick GE et al. Altered fatty acid composition of dopaminergic neurons expressing $\alpha$-synuclein and human brains with $\alpha$-synucleinopathies. J. Biol. Chem. 278, 49874-49881 (2003).

61. Shioda N, Yabuki Y, Kobayashi Y et al. FABP3 protein promotes $\alpha$-Synuclein oligomerization associated with 1 methyl-1,2,3,6-tetrahydropiridine induced neurotoxicity. J. Biol. Chem. 289(27), 18957-18965 (2014).

62. Zimmer L, Delion-Vancassel S, Durand G et al. Modification of dopamine neurotransmission in the nucleus accumbens of rats deficient in n- 3 polyunsatured fatty acid. J. Lipid Res. 41, 32-40 (2000).

63. Eusebi P, Giannandrea D, Biscetti L et al. Diagnostic utility of cerebrospinal fluid $\alpha$-synuclein in Parkinson's disease: a systematic review and meta-analysis. Mov. Disord. 32, 1389-1400 (2017).

64. Parnetti L, Castrioto A, Chiasserini D et al. Cerebrospinal fluid biomarkers in Parkinson disease. Nat. Rev. Neurol. 9, 131-140 (2013).

65. Mollenhauer B, Steinacker P, Bahn E et al. Serum heart-type fatty acid-binding protein and cerebrospinal fluid tau: marker candidates for dementia with Lewy bodies. Neurodeg. Dis. 4, 366-375 (2007).

66. Wada-Isoe K, Imamura K, Kitamaya M et al. Serum heart-fatty acid binding protein levels in patients with Lewy body disease. J. Neurol. Sci. 266, 20-24 (2008).

67. Teunissen CE, Veerhuisa R, De Vente J et al. Brain-specific fatty acid-binding protein is elevated in serum of patients with dementia-related diseases. Eur. J. Neurol. 18 865-871 (2011).

-• One of the few works on the diagnostic value of FABP3 in plasma. FABP3 could be a more suitable biomarker because of the possibility of its detection not only in cerebrospinal fluid but also in plasma.

68. Backstrom DC, Eriksson Domellof M, Linder J et al. Cerebrospinal fluid patterns and the risk of future dementia in early, incident Parkinson disease. JAMA Neurol. doi:10.1001/jamaneurol.2015.1449 (2015) (Epub ahead of print).

69. Chiasserini D, Biscetti L, Eusebi P et al. Differential role of CSF fatty acid binding protein 3, $\alpha$-synuclein, and Alzheimer's disease core biomarkers in Lewy body disorders and Alzheimer's dementia. Alzheimers Res. Ther. 9, 52 (2017).

-. Stresses on the important added value of FABP3 in differentiating between Alzheimer's disease and dementia with Lewy body patients. 
